\title{
Interactive Visual Analysis of Hierarchical Enterprise Data
}

\author{
Yu-Hsuan Chan \\ University of California, Davis \\ Email: chany@cs.ucdavis.edu
}

\author{
Kimberly Keeton \\ Hewlett-Packard Labs \\ Email: kimberly.keeton@hp.com
}

\author{
Kwan-Liu Ma \\ University of California, Davis \\ Email:ma@cs.ucdavis.edu
}

\begin{abstract}
In this paper, we present an interactive visual technique for analyzing and understanding hierarchical data, which we have applied to analyzing a corpus of technical reports produced by a corporate research laboratory. The analysis begins by selecting a known entity, such as a topic, a report, or a person, and then incrementally adds other entities to the graph based on known relations. As this bottomup knowledge building process proceeds, meaningful graph structure may appear and reveal previously unknown relations. The ontology of the data, which represents the types of entities in the data and all possible relations among them, is displayed as a guide to the analyst in the process. The analyst may interact with the ontology graph or the data graph directly. In addition, we provide a set of filtering, searching, and abstraction methods for the analyst to manage the complexity of the graph. In contrast to a top-down approach, which usually starts with an overview of the whole data set for exploration, a bottom-up approach is generally more efficient, because it often only touches a very small fraction of the data. We present several case studies to demonstrate the efficacy of this interactive graph-based analysis technique for both intra- and inter-hierarchy analysis.
\end{abstract}

Keywords-Visual Analytics, Social networks, Knowledge Management, Business Intelligence

\section{INTRODUCTION}

The desire to understand how people interact, relative to the content of their interactions, arises in many contexts. For instance, we may want to understand who is emailing whom about a particular set of topics or to understand who comments vocally on blog posts about particular topics. Other examples include understanding collaboration patterns in writing technical reports or developing source code.

These applications possess two logical hierarchies: a content-based hierarchy and a people-oriented hierarchy. Each hierarchy possesses multiple levels, which correspond to an aggregation of the adjacent lower level. For example, email messages may be aggregated into threads, which may be clustered together based on common themes. An organization's organization chart describes the hierarchy of people belonging to the organization. Relationships exist between levels of the hierarchies. In particular, for a given level in each hierarchy, multiple types of relationships may be meaningful. For instance, we can consider both sender and receiver relationships for email messages. In addition, different relationships may exist at different levels of the hierarchy. For example, if the content hierarchy represents documents and their content, people may own copies of the document, whereas they may be authors of the content.

Users want to ask a variety of questions in this space. They want to see summarized views of the hierarchies, to understand which entities are most important. They want to ask questions about semantically meaningful subsets of the hierarchies: interactions on a particular set of topics, or the contributions of an organizational unit. They may even want to compare the relationships at different points in time.

Enterprise data can present additional challenges. The geographic distribution of global enterprises means that information may be replicated and distributed across different data centers, leading to data integration challenges. Additionally, the organizational structure of an enterprise can be very dynamic. A corporation may have undergone several reorganizations over time, and an organizational unit may be renamed, merged with others, removed, or assigned to different functionalities. Such organizational dynamics present another challenge to analysis.

Understanding the complex relations embedded in enterprise data thus requires advanced analysis techniques beyond what conventional database query methods can offer. We have developed visualization-directed analysis techniques for making sense of network data. Most existing visualizations for social network analysis employ a top-down approach, providing structural overviews of the entire network to apply Shneiderman's Visual Information-Seeking Mantra [1], "overview first, zoom and filter, then detailson-demand." However, in some cases the analyst is not interested in a global view of the whole data set, but rather wants to find specific information based on a known subject or event. In these cases the user has a specific question, and a bottom-up approach may be more suitable. The analysis becomes a knowledge building process, where the analyst begins by selecting a known entity such as a topic, a report, or a person, and then incrementally adds other entities based on known relationships. As the process proceeds, meaningful graph structure may appear and reveal previously unknown relations.

In this paper, we present an interactive visual technique for analyzing and understanding hierarchical data. To demonstrate and evaluate the efficacy of the technique, we use several case studies based on technical reports produced by a corporate research laboratory. The resulting 
visualizations show sets of relevant documents or people, ordered by relevance and organized by attributes (e.g., topics or time), and facilitate navigation of different sets of related results.

\section{RELATED WORK}

\section{A. Social Network Analysis}

Many aspects of network visualization and social network analysis are relevant to our work, which is mainly about the visual exploration of relationships that exist among inherently hierarchical data sets. One prominent type of hierarchies we consider in our work is people networks. Understanding social networks and their relationships with, for example, people performance or purchasing trends, is currently of strong interest in many areas, such as the study of software developer networks and software evolution [2], [3], [4]. Typical social network analysis uses mathematical graph theories and linkage mining to characterize the structural properties of networks and understand the dynamic behavior of systems built upon them. It aims at many tasks, including centrality evaluation [5], network modeling [6], community finding [7], and link prediction [8]. More comprehensive reviews can be found in [9], [10], [11] and at the INSNA website [12]. Our work considers some of these measures for filtering and ranking operations.

\section{B. Visualizing Hierarchical Data}

Hierarchies are one of the most commonly used information structures. Over the last twenty years, there has been much research on effective display and interaction with a homogeneous hierarchy. Common ways to graphically represent a hierarchy are a treemap and a node-link graph. The former shows how an entity at higher layers of the hierarchy contains an subset of lower layer entities, and optimizes the screen space [13]. The latter explicitly shows the depth of entities, making it easy to compare the height of different layers. Fisheye [14], Hyperbolic Browser [15], H3 [16], Cone Trees [17], FSViz [18], Disk Trees [19], and many others fall into this category. However, it deals with only a single hierarchy and focuses on the efficient use of screen space, the readability, and the graph layout.

Visualizing multiple hierarchies has been studied as an enterprise wide problem in recent years. Time Tube [19] examines a single hierarchy changing over time and highlights changes. However, users are forced to integrate these changes in Time Tube cognitively across time, putting a strain on short-term memory.A botanical taxonomy visualization [20] examines multiple overlapping hierarchies and highlights the correlated entities in them, and thus reveals interesting patterns. However, it does not scale well, and it shows only inter-hierarchy relations between leaf nodes. MultiTrees [21] merge multiple hierarchies that share subtrees into a directed acyclic graph, and visualizes a focal node of interest with several parent layer nodes and children layer nodes. However it works only for hierarchies that share large enough common sub-trees. Similarly, Polyarchy Visualization [22] adresses multiple intersecting hierarchies that share at least one node, rather than sharing sub-trees. In both [21] and [22], the hierarchies are homogeneous ones, meaning that nodes in the different hierarchies are of the same type, but organized differently. Binary Tanglegrams [23] is an intuitive representation to show the relationship between two side-by-side binary trees. Nöllenburg et al. [24] experiment with several heuristics on the tanglegram layout problem. Following that, the authors apply edge bundling to tanglegrams [25], in which bundled edges show inter-hierarchy relationships with higher readability. However, the representation suffers from edge crossings, especially for flat trees of large width.

\section{Non-overview Visualization}

In some cases users are more interested in local critical areas in the graph, instead of the overall graph topology. Therefore, providing a holistic view of the entire graph, like Shneiderman's Visual Information-Seeking Mantra [1], "overview first, zoom and filter, then details-on-demand," is not best for answering their specific questions.

Some previous work starts the navigation from a focal node of interest, and then explores the surrounding subgraph of adjacent entities that are related to the current focus, as the idea "plant a seed and watch it grow" in [26]. Touchgraph [27], Palantir [28], Treeplus [26], and "search, show context, expand on demand" [29] fall into this category.

Other work starts by computing the importance of each subgraph followed by removing those less relevant subgraphs, and then visualizes the remaining graph. SocialAction [30] uses attribute ranking and coordinated views to allow users to define metrics of interest for identifying important nodes, clusters and outliers in graphs. NodeXL [31] also follows this computational approach by integrating the statistical and visual capabilities of Excel with graph visualizations. These approaches allow users to target specific features when exploring, but the computational complexity of the metrics prohibits application to large graphs.

\section{TECHNICAL APPROACH}

Our objective is to develop a visual-based query method for finding hidden or complex relations that are difficult to identify with text-based queries. Visualization also makes it much easier for the user to "see" any structure, pattern, or trend in the returned results. Although the popular top-down, overview-to-detail visualization methods are usable, they are not the most efficient way to answer specific questions about a particular topic, document, or employee. We thus advocate a bottom-up approach to visual query construction. That is, an answer is obtained by incrementally building up a query from a known subject of interest and based on visual 
feedback that shows either a partial answer or a suggestion - just enough for the user to expand the current query.

In contrast to the top-down approach, which usually starts with an overview of the whole data set for exploration, the bottom-up method only touches a small fraction of the data set, so it is generally more efficient. The analysis generally starts by presenting to the user the information she already knows. By subsequently adding a little bit of new information at a time, the user should not be overwhelmed by too much unrelated information (as often shown in an overview graph), nor confused by any abstraction done automatically to reduce clutter in the graph. While such a bottom-up approach is not new, the need to analyze data that contains multiple hierarchies with heterogeneous data demands further study and evaluation.

Our design uses the ontology of the data graph, which may contain multiple hierarchies. Because the ontology graph is generally small (e.g., only a few nodes), it provides the user a succinct view of the data, through which the user selects the entities and relations to examine. In the analysis process, users repeatedly add or remove entities or relations until they can make sense of the resulting visualization. As such, the development of interactive filtering, abstraction, aggregation, and linking operations is also essential to more effective sense making.

\section{An ENTERPRise DATA SET}

The data set used in our study is obtained from a corporate research lab. It contains information about technical reports produced in that lab from 1999 to 2008. It also contains information about the authors of the technical reports and the organization each author was affiliated with while producing a particular report. Two hierarchies are used to store this collection of information. The content hierarchy is organized into three levels: topically related groups of documents (GRP), unique documents (DOC, where a unique document may have multiple copies), and copies of documents (CPY, e.g., different copies of the same report may reside on different people's laptops). In our study, we ignore multiple copies of the same report (i.e., the CPY level). The people hierarchy is essentially an organization chart, including laboratories (LAB), departments (DEP), and persons (PSN). Note that there is a dummy root node on top of each hierarchy, ROOTPPL and ROOTDOC, respectively. Figure 1 shows the ontology of the data set. The link between PSN and DOC connects the people and content hierarchies, representing the authorship of the technical reports.

Reports are organized into twenty groups based on the topics they contain, using basic syntactic analysis and clustering precomputed by the CLUTO clustering library [32]. Thus, reports with similar contents are in the same group. Based on this analysis, each group has two sets of terms: descriptive (DES) terms summarizing the key content of

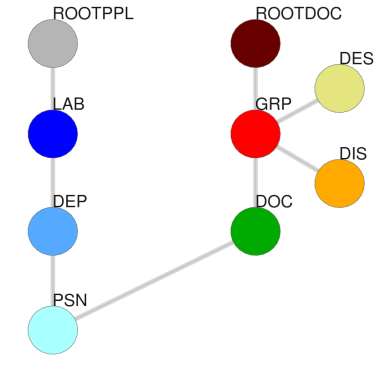

Figure 1. Ontology graph of the people hierarchy and the content hierarchy.

documents in the group, and discriminating (DIS) terms, which differentiate the group from other groups.

Authorship information, including author names and affiliations at the time a technical report is published, is reported by the corporate research library for each of the technical reports in the data set. Authors' affiliations may change over time, due to job transfers or corporate reorganizations. In order to present a single people hierarchy, we processed the data to associate each author with the department and laboratory to which their latest technical report is attached. This most closely approximates the current people hierarchy, with the caveat that employees who are no longer with the company are affiliated with their last organization (which may no longer exist in the current organization chart).

The author and affiliation information provided by the corporate research library does not always allow us to perfectly disambiguate individuals or organizations, however. Ambiguity in a name can arise from variations in how an entity is referenced (e.g., University of California at Davis vs. UC Davis) or from the existence of several entities with the same name (e.g., George Bush and George W. Bush), or even from spelling mistakes in the name (e.g., Kimberly vs. Kimberley). As a result of this ambiguity, our data processing occasionally produced duplicates of individuals or organizational units in the data set, and the relations between such duplicates are not explicitly defined, potentially leading to confusing visualizations. However, as shown in our case studies, during interactive visual analysis, one can easily identify duplicates in the data. Our system provides operations to merge duplicates into a single node.

\section{Case Studies}

In this section, we present three case studies on this enterprise data set to show how interactive visual analysis can be helpful to reveal trends, patterns, and information that conventional database queries do not provide.

\section{A. Person-Oriented Analysis}

In the first case study, we consider a particular employee of interest and see how this person worked within the organization and collaborated with others. 
1) Publications of an individual: We start by selecting the PSN node from the ontology graph, and enter the name of the individual "BlindedPersonName" as keyword for searching. (In all graphs of this paper, we hide names of individuals for privacy reasons.) This gives us twelve PSN nodes scattered in the display space. They are duplicates of the same employee. Next, we select the relation between PSN and DEP, followed by adding relations between DEP, LAB, and ROOTPPL nodes. Figure 2(a) displays the resulting graph, in which we see the employee's department and laboratory affiliations. As new nodes are added to the graph, its force-directed layout is redone, placing all nodes around the ROOTPPL node.

The graph becomes somewhat cluttered, so we merge LAB nodes (in dark blue) with identical names, all of which are represented by a squared node, as shown in Figure 2(b). Note that the corresponding merged edges are drawn thicker. We can now better see the relationships between the LAB, DEP, and PSN nodes.

The presence of duplicate individuals illustrates several insights about the data set. Duplicate PSNs linking to the same DEP/LAB nodes likely indicate variations in the person's name. Duplicates with different DEPs but the same LAB may indicate that the department has been renamed, or that the individual switched groups within the lab. Duplicates with different DEPs and different LABs indicate either a reorganization or a transfer of the individual to a new lab. Accordingly, the organizational changes can be identified in Figure 2(a)(b) by observing the intra-hierarchy relations between the duplicates of an individual and the the higher layer nodes of organizational units.

To examine information in the content hierarchy related to BlindedPersonName, we add DOC nodes (in green) to the current graph. Figure 2(c) shows the result where all DOC nodes and other nodes are centered around the collapsed LAB nodes. Next we add GRP nodes in red and relayout. The resulting graph becomes GRP-centered instead of LAB-centered, such that we can better see how many technical reports are in each group, as shown in Figure 2(d). To continue our analysis by adding other information, we collapse all DOC nodes to GRP nodes to free some space. The new triangular nodes in Figure 2(e), to which PSN nodes directly connect, represent the collapsed results, allowing us to see PSN and GRP relations. We find five PSN nodes contribute to GRP node \#13 only, five PSN nodes contribute on to GRP node \#8 only, and two PSN nodes contribute to all three GRP nodes (\#7, \#8, and \#13).

Finally, to find out what these groups of technical reports are about, we add DIS nodes (in orange) to the graph. We also remove the DEP and LAB nodes to make the graph even more GRP-centered. The resulting graph is shown in Figure 2(f), from which we can see that the three groups share ideas and interests. In particular, two particular occurrences of BlidedPersonName are clearly associated to those discriminating term nodes (DIS) in the middle, as a result of the force-directed layout. This sequence demonstrates how our interactive system enables the user to incrementally build up knowledge about an entity and manage complexity with simple abstraction methods. One interesting next step is to encode time information into the visualization, which is covered in the third case study.

2) Co-Authorship: After seeing BlindedPersonName's publications, now we want to look at how this individual collaborated with others. To start from the result in Figure 2(e), we merge these twelve PSN nodes since they are duplicates of BlindedPersonName, expand collapsed GRP nodes to show DOC nodes, and remove DEP and LAB nodes to show only this individual and all his documents, as shown in Figure 2(g). Then we select the relation between DOC and PSN in the ontology to include all co-authors that also contributed to these documents, as shown in Figure 2(h). However, the redundancy of individuals remains for these newly included PSN nodes. Therefore we label these blue PSN nodes with their names to identify duplicates, and merge duplicates if any. The initial clean-up result is in Figure 2(i), in which merged PSN nodes are in squared shape. After merging duplicates of co-authors, the graph is still quite complicated, because of the edge crossings around co-authors that frequently collaborated with BlindedPersonName, as indicated by the multiple documents they share with BlindedPersonName in the graph. To highlight these important collaborators, we compute the degree of the nodes in the graph, and filter out those co-authors with low degree. The degree of a node is the number of edges that link to or from this node. After filtering, the number of collaborators becomes more manageable, as shown in Figure 2(j). We re-layout these PSN and DOC nodes by putting people who share documents closer to each other. DOC nodes are labeled by keywords to reveal the topics of the collaboration, as show in Figure 2(k). The keyword labels of the green DOC nodes show that these important collaborators mostly worked with BlindedPersonName on the following topics:

- Performance: performance evaluation, performance analysis, and performance models.

- Resources: system's resource requirements, resource usage optimization, shared resource pools, and capacity management.

- Others: case studies, web differentiated services, and admission control.

Another alternative for visually organizing these collaborations is to make use of GRP nodes, as shown in Figure 2(l), and to switch to a content-oriented graph. BlindedPersonName is the square node in the center with the dark outline. These important collaborators are pulled to GRP nodes indirectly by DOC nodes they co-authored. We see that these collaborative documents fall in two clusters (GRP \#8 at the 


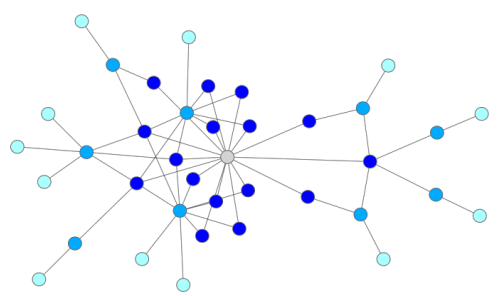

(a)

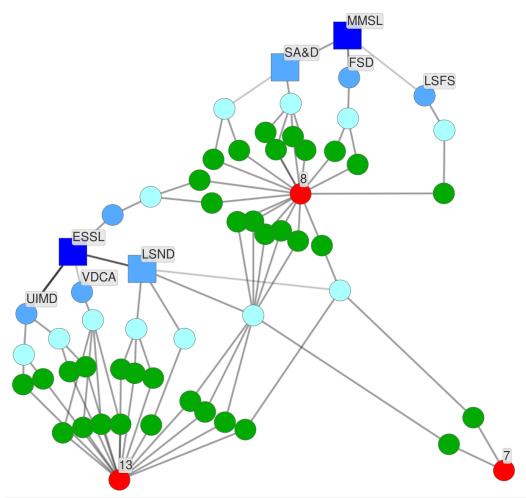

(d)

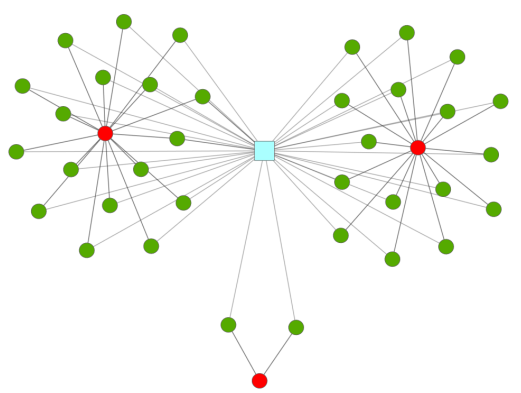

(g)

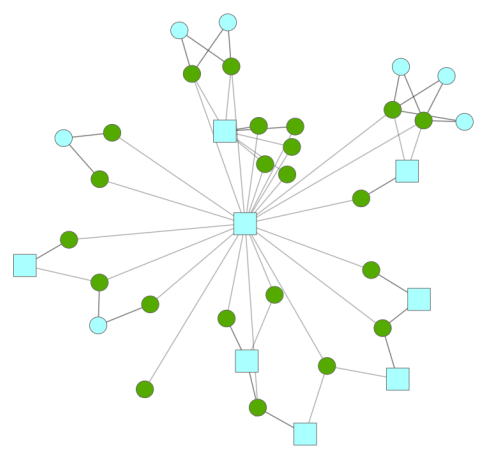

(j)

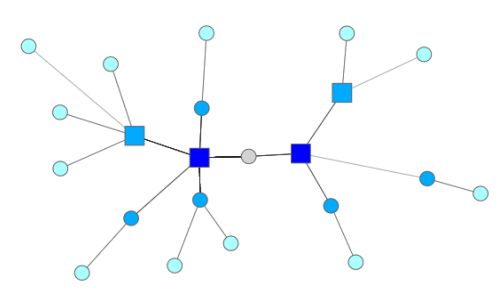

(b)

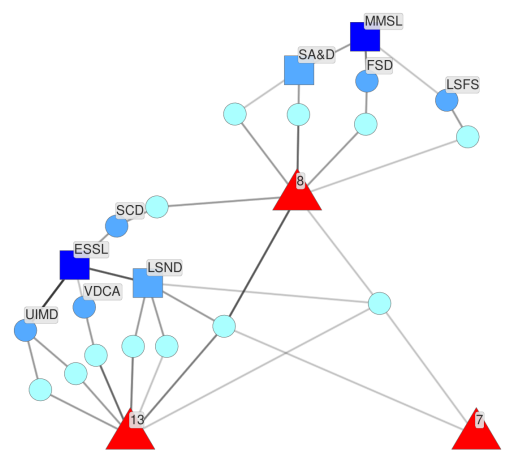

(e)

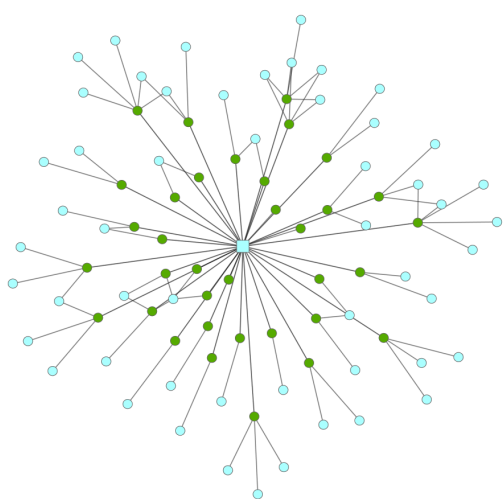

(h)

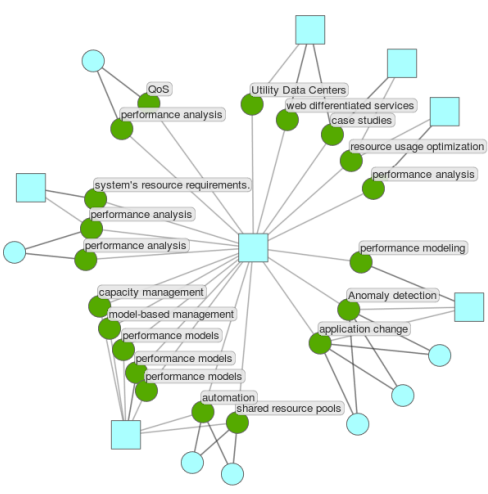

(k)

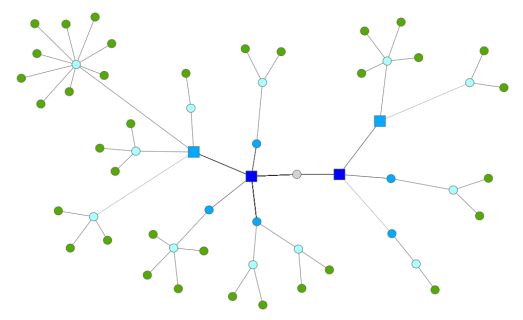

(c)

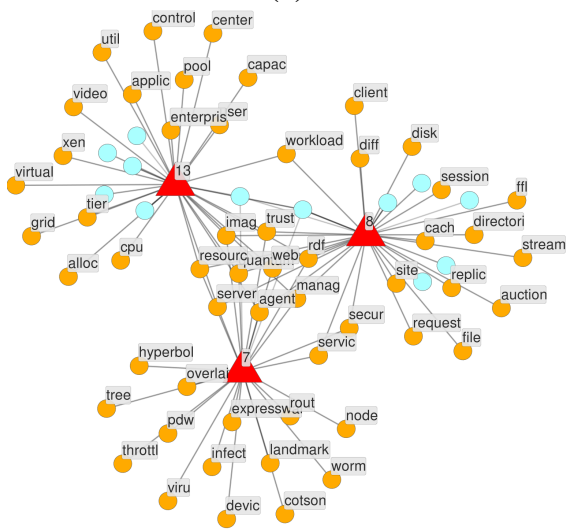

(f)

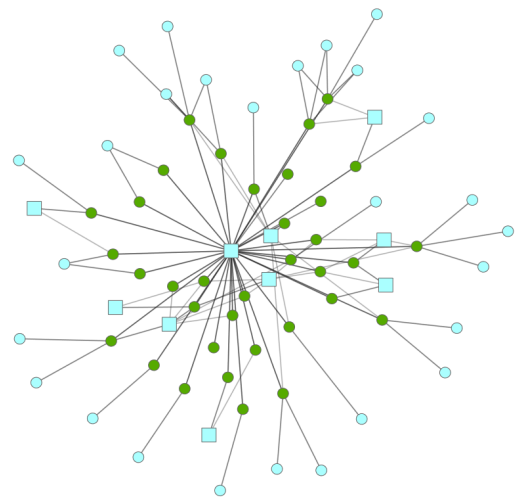

(i)

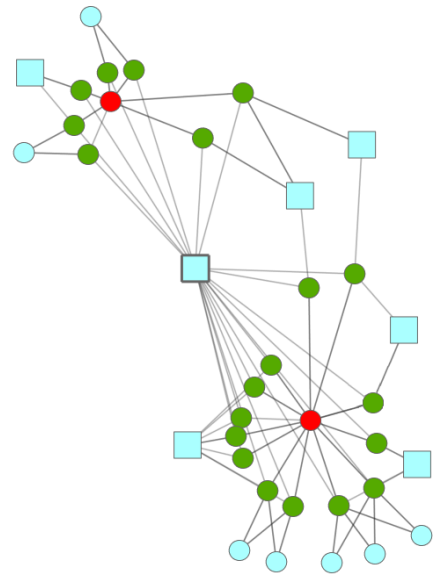

(l)

Figure 2. Person-oriented analysis. (a)-(f): Publications of an individual. (g)-(l): Co-authorship. Node colors are the same as in Figure 1. Analysis begins by searching for BlindedPersonName, resulting in duplicates of the person, labs and departments in (a). Merging nodes with duplicate names into square nodes in (b) simplifies layout. Documents nodes in green are included in (c), and GRP nodes in red in (d). Abstraction is achieved by merging DOC nodes into their parent GRP nodes, indicated by traingular nodes in (e). Discriminating terms for the GRPS are then added in (f). BlindedPersonName's DOCs are organized by GRPs in red in (g). Co-authors are added in (h), with duplicates of co-author PSN nodes merged in (i) and infrequent collaborators removed in (j). Collaboration topic labels are shown in (k). Frequent co-authors are organized by GRPs in (l). 
top left and \#13 at bottom right). Some collaborators worked on only one of the clusters, while others contributed to both clusters (e.g., the two square nodes at the top right in the middle of the two GRP nodes).

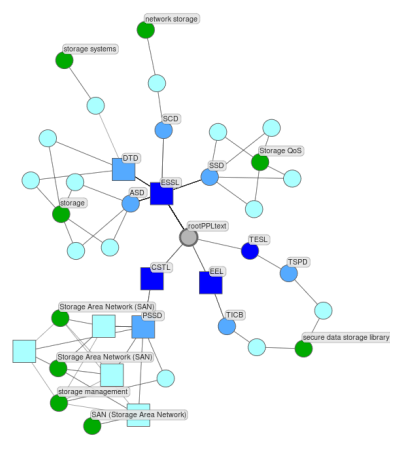

(a)

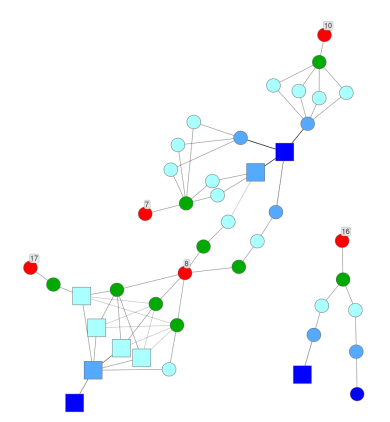

(b)

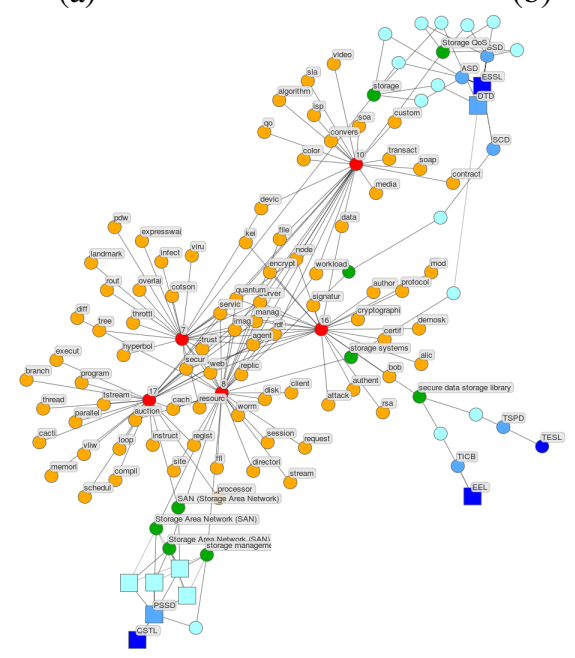

(c)

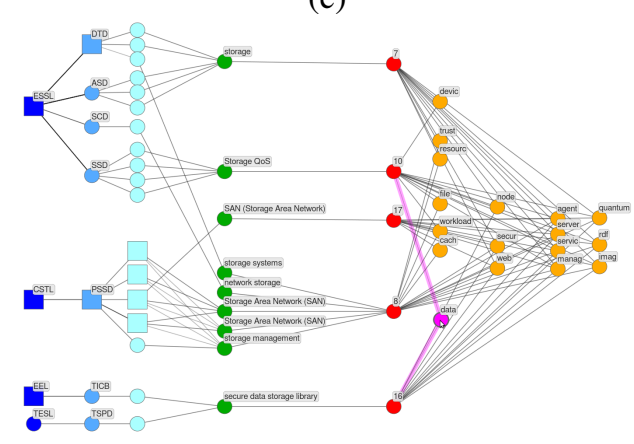

(d)

Figure 3. Content-oriented analysis, searching for "storage." (a) Documents (DOCs in green) about "storage," with their authors (PSNs in light blue) and affiliations (DEPs in blue and LABs in dark blue). (b) Graph reorganized by clusters (GRPs in red) at a higher layer in the content hierarchy. (c) Discriminating terms (DISs in orange) that shows semantic information. (d) Revised layout results in a bipartite-like graph.

\section{B. Content-Oriented Analysis}

Users may want to start the exploration from the content rather than an individual. For example, users may have some topics of interest in mind, or they may want to find all technical documents associated with a product. This exploration is especially useful for electronic discovery, to quickly identify all of the documents that are potentially relevant to a legal case, as well as all individuals who contributed to these documents, so they can be interviewed.

We start by searching documents that have the keyword "storage," and it results in nine DOC nodes in green for this topic. Next, we select the relations in the ontology graph to incorporate nodes into the graph. We start from the link from DOC to PSN in light blue, followed by DEP in blue, LAB in deep blue, and finally ROOTPPL in gray, in order to reveal the people hierarchy about the "storage" topic. Figure 3(a) displays the resulting graph, and shows that authors contribute to this topic are from four LAB nodes in deep blue: ESSL, CSTL, EEL and TESL. Note that there is a strong intra-lab collaboration in CSTL lab at the bottom left, where four square PSN nodes and the four documents they co-author are strongly connected. In this graph, we can see who works on documents about a particular topic, and which organizational units they belong to.

With the ontology graph, users can choose to look at higher layers of hierarchies to abstract the details. Therefore at this point we decide to navigate up a layer in the content hierarchy to gain more insight about this topic. This time, we select the relation between DOC and GRP (in red) to show clusters, and remove the root node of the people hierarchy to relax the ties between LAB nodes, as shown in Figure 3(b). Then we select the relation between GRP and DIS (discriminating terms of clusters, in orange) to show the semantic meanings of the clusters, as shown in Figure 3(c). This graph is a little overwhelming because all 25 DIS nodes of a GRP node are shown. We can avoid this by showing only those DIS nodes shared by multiple GRP nodes after filtering out DIS nodes with low degree.

Then we manipulate the node positions according to the nodes' layers in the hierarchy, as shown in Figure 3(d). This gives a bipartite-graph-like view between DOC nodes related to "storage" and the PSN nodes of their authors, along with higher layers of the hierarchies aside for visual abstraction. By DEP and LAB nodes at the left, we know what organizational units have worked on this topic. By GRP and DIS nodes with labels at the right, users can quickly get a sense about the content of these documents.

In this case study, we have shown that with the ontology defining both intra-hierarchy and inter-hierarchy relations, users are able to explore the large data set easily and quickly. It guides users by suggesting the next possible relations and entities to examine, and facilitates exploration within the same hierarchy, as well as between hierarchies. When we 
switch to higher layers in hierarchies for abstraction, the contextual information of the original lower layers remains, and thus mitigates the loss of information.

\section{Temporal Domain Analysis}

In this case study, we are interested in what research topics this corporate research lab has worked on over the years, and how research topics changed over time. Therefore we compare GRP nodes between different time periods, since GRP nodes can be seen as a content-based classification of all documents.

To incorporate the time information, we use a scatter plot with line segments to visualize the distribution of groups of documents over time, as shown in Figure 4. Each line represents a GRP node in the data graph. The documents in that group are binned according to publication year. There is an interactive histogram on the left of the scatter plot that shows the total number of documents of each group. Hovering over the histogram, the line of the corresponding group is highlighted. In this interface, users can hover over these bars to quickly glance at the distribution of the documents over time in that group, and efficiently compare different groups.

We observe two types of trends in research topics over time, as show in the two views in Figures 4, in which the lines of the document groups with the trend are highlighted:

1) Hot topics: the two highlighted lines at the top view illustrate two sets of hot topics (i.e., a large number of reports on a particular topic in a particular year). A peak in an early year indicates that the topic has become less popular in recent years, while a peak in a recent year means that it is an emerging topic.

2) Ongoing studies: two sets of reports are illustrated by the highlighted wave-like lines at the bottom view. A steady number of reports over the years indicates an ongoing research effort.

This interactive time-line view is coupled with the data graph to enable users to filter documents by time. Clicking on any node on a line in this graph, the corresponding documents that belong to this group and are published in this year is highlighted. Clicking on any bar in the histogram highlights the corresponding GRP node in the data graph. The semantic entities, such as DIS and DES, can be used to verify the content of the GRP lines in this time-line view. Then we can use the ontology graph to further query about the authors of these documents, and the organizational units of the authors.

\section{Discussion AND Future Work}

Several aspects of our current system can be enhanced. First, it would be helpful to provide views of the data based on certain statistical or importance measures of the data. Second, our current system does not provide any support for tracking the steps the user has taken to derive a particular

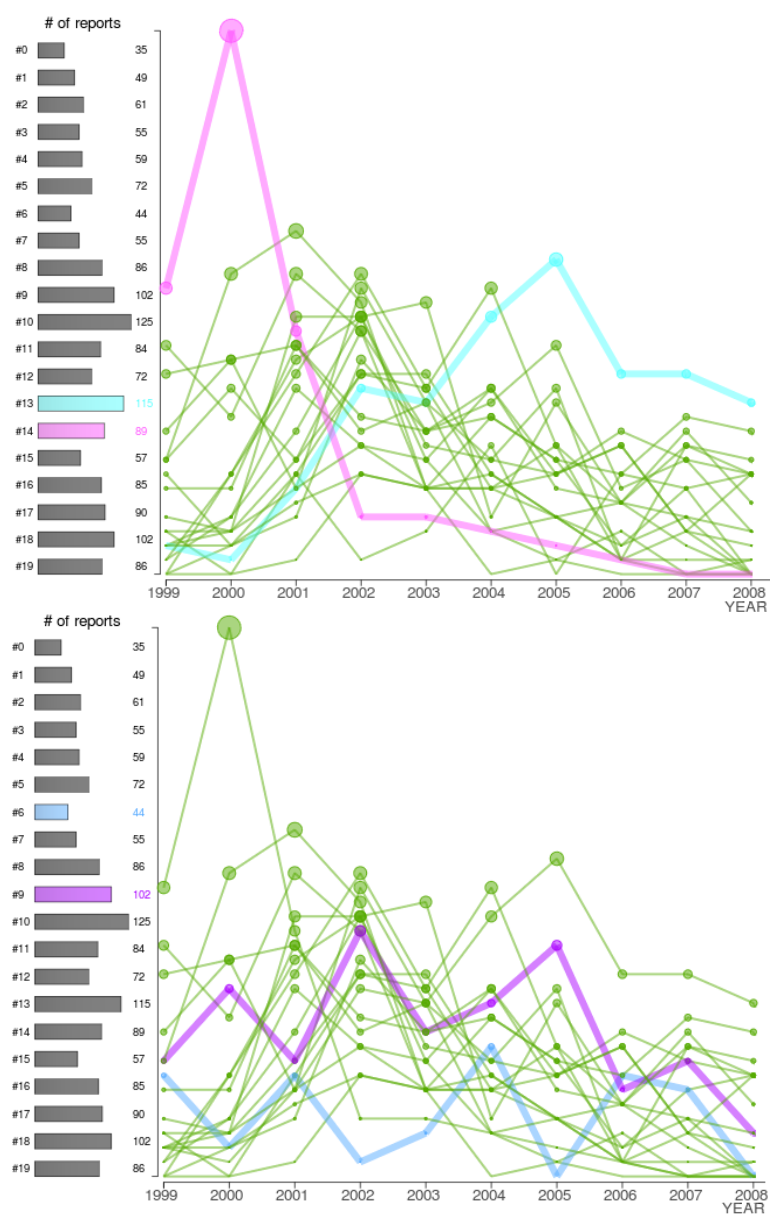

Figure 4. Temporal domain analysis of the trends in research topics over time. Each line represents a clustered group (GRP) of documents. The Xaxis is the year, and the y-axis is the total number of documents in the group published that year. The upper view shows both hot topics, and the bottom view shows ongoing studies.

finding. In data exploration, users may want to preserve intermediate results and findings, and then review them later or share them with others. That is, future analyses could be based on the history of a previous analysis coupled with undo and redo operations. A history visualization would also be desirable. We would also like to add support for semantic filtering. We plan to experiment with simple thresholdbased techniques, such as top $\mathrm{n} \%$ contributors or top $\mathrm{x} \%$ contribution, to filter a selected dimension of a hierarchy, as well as more semantically rich criteria, such as keywords that describe the topics associated with each content node.

Finally, we would also like to study how to effectively support both top-down exploration and bottom-up knowledge building in one visual analysis framework, such that the user can freely switch between them or use them simultaneously. We would like to conduct a thorough user study to qualitatively and quantitatively compare the topdown and the bottom-up approaches. 


\section{CONCLUSION}

The successes of an enterprise largely rely on its ability to make critical business decisions by effectively utilizing the vast amounts of information acquired from diverse sources. Traditional information management and knowledge discovery tools fail to cope with the information explosion facing many major enterprises. In this paper, we demonstrate that visualization is a promising solution for this pressing problem. Our bottom-up approach effectively supports goaloriented, interactive analysis of enterprise data in terms of a people hierarchy and a content hierarchy.

The contributions of this work are summarized as follows:

1) We perform a preliminary study on a bottom-up approach for exploring a data set with multiple hierarchies. This method starts with a known subject or entity of interest and then visually adds relations and other entities, in order to derive the answer we are looking for, or simply to explore the data. An ontology graph facilitates the addition of entities. The primary benefits of this approach in comparison with a top-down approach include: answering specific questions more efficiently, avoiding confusing users with extraneous nodes, and reducing clutter in the graph by abstraction.

2) We provide techniques for interactive operations on multiple hierarchies, such as searching, filtering, and aggregating for abstraction, to manage the complexity of the graph.

3) We demonstrate the merits of our approach to interactive visual sense making using several case studies exploring authorship and collaboration patterns in a technical report data set from a corporate research lab.

\section{ACKNOWLEDGMENT}

This research was supported in part by the U.S. National Science Foundation through grants CCF-0938114, CCF-0808896, CNS-0716691, and CCF-1025269, the U.S. Department of Energy through the SciDAC program with Agreement No. DE-FC02-06ER25777, and HP Labs and AT\&T Labs Research.

\section{REFERENCES}

[1] B. Shneiderman, "The eyes have it: a task by data type taxonomy for information visualizations," Proc. of 1996 IEEE Symposium on Visual Languages, 1996.

[2] C. Bird et al., "Mining email social networks," in Proc. of MSR'06.

[3] M. Ogawa et al., "Visualizing social interaction in open source software projects," in Proc. of APVIS '07.

[4] M. Ogawa et al., "StarGate: A Unified, Interactive Visualization of Software Projects," in PacificVis '08.

[5] L. Freeman, "Centrality in social networks conceptual clarification," Social Networks, 1979.
[6] A.-L. Barabasi et al., "Linked: The New Science of Networks," American Journal of Physics, 2003.

[7] M. Newman, "Finding community structure in networks using the eigenvectors of matrices," Physical Review E, 2006.

[8] D. Liben-Nowell et al., "The link-prediction problem for social networks," Journal of the American Society for Information Science and Technology, 2007.

[9] S. Wasserman et al., Social network analysis.

[10] J. P. Scott, Social Network Analysis: A Handbook.

[11] M. Huisman et al., Software for social network analysis.

[12] INSNA. http://www.insna.org/

[13] Treemaps for space-constrained visualization of hierarchies. http://www.cs.umd.edu/hcil/treemap-history

[14] G. W. Furnas, "Generalized fisheye views," $A C M$ SIGCHI Bulletin, 1986.

[15] J. Lamping et al., "Laying out and visualizing large trees using a hyperbolic space," in Proc. of UIST '94.

[16] T. Munzner, "Exploring large graphs in 3D hyperbolic space," IEEE Comput. Graphics and Appl., 1998.

[17] G. Robertson et al., "Cone trees: animated 3D visualizations of hierarchical information," in $\mathrm{CHI}$ ' 91 .

[18] J. Carriere et al., "Interacting with huge hierarchies: Beyond cone trees," in Proc. of InfoVis '95.

[19] E. H. Chi et al., "Visualizing the evolution of Web ecologies," in Proc. of CHI'98.

[20] M. Graham et al., "A comparison of set-based and graph-based visualisations of overlapping classification hierarchies," in Proc. of AVI 'OO.

[21] G. W. Furnas et al., "Multitrees: enriching and reusing hierarchical structure," in Proc. of CHI '94.

[22] G. Robertson et al., "Polyarchy visualization: visualizing multiple intersecting hierarchies," in $\mathrm{CHI}$ 'O2.

[23] N. Sheth et al., "Treemap, Radial Tree, and 3D Tree Visualizations,' in Proc. of InfoVis '03.

[24] M. Nöllenburg et al., "Drawing binary tanglegrams: An experimental evaluation," in Proc. of ALENEX '09.

[25] D. Holten et al., "Visual Comparison of Hierarchically Organized Data," Computer Graphics Forum, 2008.

[26] B. Lee et al., "TreePlus: interactive exploration of networks with enhanced tree layouts," IEEE Trans. Vis. Comput. Graphics, 2006.

[27] TouchGraph. http://www.touchgraph.com

[28] Palantir. http://www.palantirtech.com

[29] F. van Ham et al., "Search, show context, expand on demand: supporting large graph exploration with degreeof-interest." IEEE Trans. Vis. Comput. Graphics, 2009.

[30] A. Perer et al., "Balancing systematic and flexible exploration of social networks." IEEE Trans. Vis. Comput. Graphics, 2006.

[31] M. A. Smith et al., "Analyzing (social media) networks with NodeXL," in Proc. of C\&T '09.

[32] CLUTO. http://glaros.dtc.umn.edu/gkhome/views/cluto/ 